\title{
Design of On-board Xenon Warning Lamp Based on Modular Control Su Yi
}

\author{
Wuxi Vocational Institute of Commerce, Wuxi, Jiangsu 214153, China \\ email: 402814799@qq.com
}

Keywords: Modular control, Xenon lamp; Warning

\begin{abstract}
On-board xenon lamp (HID) has a series of advantages such as high luminous efficiency, high brightness, low energy consumption, high reliability, being not affected by on-board voltage fluctuation and long service life; especially in terms of the use of warning lamps, it has such advantages compared to halogen lamps and LED lamps and lanterns. This paper mainly studies the design of an on-board xenon warning lamp based on modular control, which has a good warning effect on automobile traffic safety.
\end{abstract}

\section{Background}

By the end of 2017, there were more than 310 million vehicles in China, and 28.8 million new vehicles entered into the market in 2017. In 2017, about 63,000 deaths were arisen from traffic accidents. Relatively speaking, traffic accidents caused by bad weather will increase significantly, causing heavy losses to the society and individuals.

At present due to the improvement of traffic lighting conditions, the use of fog lamps is relatively small, but there is a need for vehicles to be equipped with lights for long distance warning in order to make the safe operation of the vehicles in bad weathers. The existing technology is mainly based on yellow light, which has a good long-distance warning effect. A yellow lampshade is configured with xenon lamp (HID) as light source, which can achieve this purpose.

\section{Overall Design Principle.}

In this paper, a common long-row one-piece on-board warning lamp was improved to be a separate on-board warning lamp, which is controlled by means of modularization. The on-board warning lamp mainly include a controller assembly A, cable connector B and xenon flash lamp C. For the convenience of layout, most of the components are placed into the controller assembly $\mathrm{A}$, and the xenon flash lamp C contains xenon lamp tube and accessory components, as shown in figure 1 :

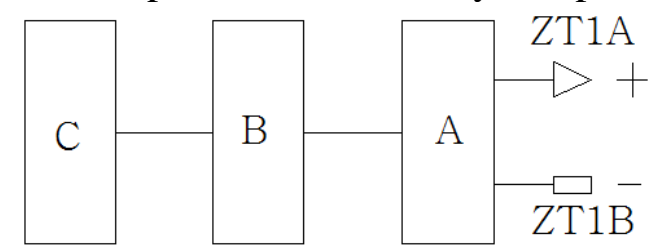

FIG. 1 General structure diagram of the warning lamp

This design application on a small manned vehicle can be installed by temporary mode, and a magnetic adsorption device with damping sucker is installed at the bottom of the xenon flash lamp C; it is placed on the proper position of the vehicle ahead of schedule, wireless signal is transmitted to the controller assembly $\mathrm{A}$, and the controller assembly is connected with the power supply for use, and it can be dismantled after using it; If it is used on medium and large vehicles and small vessels, this can be set on a special warning position.

This design can achieve the following objectives: 1 . Do not change the operation interface of existing vehicles, but obtain the power supply relying on the on-board DC power outlet; 2 . Expand other control functions in the controller assembly A, and select more energy-saving and environment-friendly components; 3. It is convenient and quick for operators in emergency operation to achieve the purpose of emergency. 


\section{Composition of function Modules}

1. The controller assembly A comprises a power switch K1, fuse FU, fan FS, current limiting resistor R1, power indicator light green LED1, power source anti-jamming filter and voltage stabilizing circuit module DL1, AND electronic switching circuit module DL2, timing pulse generator circuit module DL3, high-voltage limiting circuit module DL4, acceleration gate switching circuit module DL5, delay trigger signal circuit module DL6, separately excited type high voltage generator circuit module DL7, flash energy control circuit DL9, GT0 high-power turn-off thyristor SCR, high voltage neon indicator light NH1, ESR high-voltage energy storage capacitor C1 and a chassis with DC plug connection ZT1A/ZT1B.

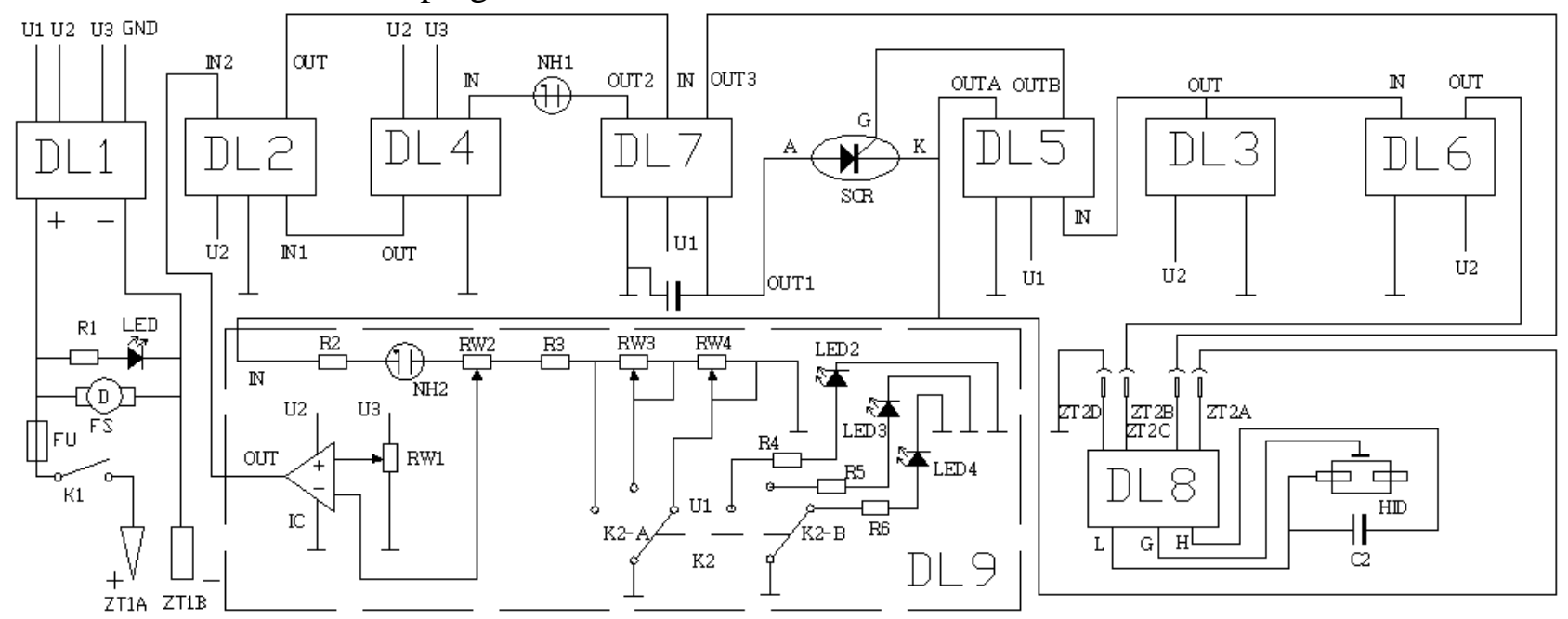

FIG. 2 Schematic diagram of warning lamp circuit

In the anti-interference filter and voltage stabilizing circuit module DL1, a voltage stabilizing integration block W4810 and power source electromagnetic compatibility type anti-interference filter component are mainly adopted.

In the AND electronic switching circuit module DL2, a two-input AND circuit composed of triode is mainly adopted.

In the timing pulse generator circuit module DL3, a time-base integration block 555 is mainly adopted, and the width of the output rectangle pulse is $5 \sim 10 \mathrm{~ms}$ and the period is $0.5 \sim 1 \mathrm{~s}$.

In the high voltage limit circuit module DL4, a comparator circuit composed of integrated operational amplifier LM358 is mainly used.

In the acceleration gate switching circuit module DL5, acceleration capacitors are mainly connected in parallel at both ends of the input resistor in the pulse switching circuit, so that the switching circuit can accelerate saturation conduction and speed up stopping each time.

In the delay trigger signal circuit module DL6, the input end of the pulse emitter output device is mainly connected to an integral capacitor, so that the output signal can be delayed by $0.2-0.5 \mathrm{~ms}$ compared with the input signal.

In the separately excited high voltage generator circuit module DL7, a 42-input NAND CD4011 integrated block is mainly adopted for the symmetric square wave generator, and IGBT field effect transistor with the radiator is also used for power transformation.

The turn-off thyristor SCR is equipped with a suitable radiator.

2. Cable connector B, with connectors ZT2A/ZT2B/ZT2C/ZT2D.

3. Xenon flash lamp $C$ is composed of xenon flash lamp circuit module DL8, xenon flash lamp tube HID, xenon flash tube base, ESR high voltage flash capacitor C2 and xenon flash lamp body, etc. A transparent flash lamp shade is installed on the lamp body of the xenon flash lamp C, including 50\% colorless transmission area and 50\% faint yellow light transmission area, and it is a tempered glass lamp shade composed of spacing square matrixes. 


\section{Specific Working Principle}

As shown in figure 2[1], the working principle is as follows: when the power switch K1 is turned on, the power supply is already on, the fan FS works, the power indicator LED is on, and the circuit is put into normal operation. At that time, the timing pulse generator circuit constantly produces rectangle narrow pulses, enabling the thyristor SCR to be opened and closed from time to time, and the delay trigger signal circuit gives out a trigger signal only every time when the thyristor SCR is closed, so that the xenon flash lamp circuit flashes once, and then the thyristor SCR will be opened again after the flashing; During the opening period of the thyristor SCR, the electric energy in the high voltage energy storage capacitor $\mathrm{C} 1$ will be charged by the flash capacitor $\mathrm{C} 2$ in the xenon flash lamp circuit by virtue of the thyristor SCR. AND electronic switching circuit needs to work on demand in order to make the separately excited high voltage generator circuit work, namely, when the high voltage generated by the separately excited high voltage generator circuit does not exceed the safety limit, and the voltage charged by the flash capacitor $\mathrm{C} 2$ in the xenon flash lamp circuit does not exceed a specified value at the same time, the two input terminals IN1 and IN2 of AND electronic switching circuit are low, the output terminal OUT of the electronic switching circuit outputs a high level to the input terminal IN of the separately excited high voltage generator, and the separately excited high voltage generator can continue to work; Whenever possible, as long as there is one of the input terminals IN1 and IN2 of AND electronic switching circuit outputs a high level due to more than the voltage limit, the AND electronic switching circuit will output a low level immediately to enable the separately excited high voltage generator circuit to stop working at once, and it will not resume normal work until there is flashing once or twice[2].

The operational logic process of xenon flash lamp is as below:

The timing pulse generator circuit continuously produces certain regular rectangular pulses, with a period of $0.5 \mathrm{~s} \sim 1 \mathrm{~s}$, of which the duration of high voltage pulse levels is $5 \sim 10 \mathrm{~ms}$, and this parameter can be set in advance and remains the same; When the rising edges of such pulses arrive, the signals flow in two ways: the first-way signals run through the acceleration gate switching circuit, so that the acceleration gate switching circuit produces a trigger current with high level at the output terminal OUTA and low level at OUTB, which can turn off the closing pathway of the thyristor SCR, that is, the high-voltage energy storage capacitor C1 in the separately excited high voltage generator stops charging the flash capacitor C2 in the xenon flash lamp circuit through the thyristor SCR; the second-way signals run through the integral delay action to make the delay trigger signal circuit's output terminal OUT output a trigger signal, which lags $0.2 \sim 0.5 \mathrm{~ms}$ from the rising edges of the pulses, to the xenon flash lamp circuit, so that the xenon flash lamp is triggered to flash for about $0.2 \mathrm{~ms}$ and then automatically go out; at this time, the flash capacitor C2 has discharged the xenon flash lamp, and after the flashing, when the falling edge of the timing pulse comes, the acceleration gate switching circuit will produce a trigger current with high level OUTB and low level OUTA, which can open the closed thyristor SCR immediately, so that the energy storage capacitor C1 would recharge the flash capacitor C2 which has just flashed once and has been discharged in part, while waiting for the next flashing discharging. Thus, the circuit will enter into the next cycle[3].

In the flash energy control circuit DL9 mentioned, the principle of controlling the flash energy of the xenon flash lamp is to control the energy of the flash capacitor C2 discharged to the xenon flash lamp each time, and its calculation formula is below.

$\mathrm{WN}=0.5 \mathrm{C}\left(\mathrm{UN}^{2}-\mathrm{UO}^{2}\right)$.

Wherein: WN -- the rated flash energy of the xenon flash lamp, in joule (J);

$\mathrm{C}$-- the measured electric capacitance of the flash capacitor C2, in farad (F);

$\mathrm{UN}$ - the end voltage of the flash capacitor $\mathrm{C}$ at the rated flash energy of the xenon flash lamp tube HID, in volt; or V.

UO -- the extinguishing voltage after the xenon flash lamp tube HID flashes, in volt (V).

To control the flash intensity of the xenon flash lamp, as long as the charging voltage Un at two ends of the flash capacitor C2 before flashing discharge is changed, the Un is changed 
within the range of $\mathrm{UN} \leq \mathrm{Un}<\mathrm{UO}$, the change in the flash energy of the xenon flash lamp could be controlled, so that it can meet the demand of more adapting to the environmental status[4].

In this design[5], the high and low signals for controlling the charged voltage on both ends of the flash capacitor C2 are used to limit the size of the high voltage generated by the separately excited high voltage generator circuit, to control the change in the flash intensity of the xenon flash lamp. Its process is as follows: the sampling input terminal of the circuit under the control of flash energy is the positive input terminal of the flash capacitor $\mathrm{C} 2$, and it is also the output terminal of the turn-off thyristor SCR cathode K terminal; after a signal passes by the resistor R2 and the flashing neon indicator lights $\mathrm{NH} 2$ and RW2, it is taken out of the sampling output terminal that is, the active terminal of the potentiometer RW2 and it is sent to the inverting input terminal of the operational amplifier IC "-" side; when the sampling voltage reaches and is slightly higher than the same phase input terminal of the operational amplifier IC "+" side, the output terminal OUT of the IC falls to a low level immediately from a high level, leading to the output terminal OUT immediately changed by AND electronic switching circuit to a low level from the high level, so that the separately excited high voltage generator circuit stops inverter booster; after the xenon flash lamp flashes once, the sampling voltage falls once again, and the output terminal OUT of the operational amplifier IC and the output terminal OUT of the AND electronic switching circuit restore to a high level, so that the separately excited high voltage generator circuit restores its inverter function to produce high voltage.

Where adjusting the potentiometer RW2 to the active terminal to short R3, K2 is set to the "high" gear, and the xenon flash lamp has the highest flash energy, up to the rating, when regulating the potentiometer RW2 can change the light energy between the rating to two-thirds of the rating in a stepless manner; short the active terminal of the potentiometer RW2 end and R3, set K2 to the "medium" gear, adjust the trimming resistor RW3, and the flash energy is up to around two-thirds of the rating, when regulating the active terminal of the RW2 can change the flash energy in a certain range. Similarly, after shorting the active terminal of RW2 and R3, put the switch K2 to the "low" gear, adjust the trimming resistor RW4 to make the flash energy about one-third of the rating, and if regulating RW2 again, the flash energy of the xenon flash lamp can be regulated at less than a third of the rating[6]. The control switch $\mathrm{K} 2$ is suitable for the fast gear switching, while the potentiometer RW2 can be used to adjust and change the flash energy in a certain range at each gear in a stepless manner. K2.

Light emitting diode indicators are used indicate the corresponding gear positions of the switch

\section{Conclusion}

Though a wide range of LED lamp applications has been existing, on-board xenon warning lamp is still has extensive market value, and it brings play to long-distance warning effect on protecting the safety of vehicles and pedestrians where it is to be alert, which cannot be achieved by use of LED lamps or common lamps and lanterns. Adopting the design plan with the use of on-board power supply has the features, including low cost, good long-distance effect and strong adaptability and provides the safeguard for vehicle safety.

\section{References}

[1] Design of Strobe Rain and Fog Warning Lamp Device on Farm Vehicle. Journal of Agricultural Mechanization Research. Su Yi. July, 2007.

[2] Study of the Equipped Devices for One Multifunctional warning Light. Xia Bing, Xia Jiqiang, He Li, Wang Ning, Ye Wentao. Modern Chemical Research, October 2016,

[3] The Research of Vehicle-Based Warning Lightbar with High Power and Multifunction. Jin Shanhai. Master's Thesis, Yanbian University. May 2008,

[4] Design of Automotive Neon Headlight System based on IPC16C45. Peng Chunfu, Yuan Guobin. 
Software Guide, Issue 17, 2005.

[5] Research and Design of HID Headlamp Controller based on Single Chip Microcomputer. Xu Yunwu, Ouyang Mingxing, Li Yan. Automation Technology and Application. Issue 7, Volume 30, 2011.

[6] Development History and Trend of Automotive LED. Chen Lixin, Zhong Yong. Electromechanical Technology. February, 2013. 